CLINICAL STUDY

\title{
Hemodynamic instability during resection of pheochromocytoma in MEN versus non-MEN patients
}

\author{
Anouk Scholten ${ }^{1}$, Menno R Vriens ${ }^{1}$, Geert Jan E Cromheecke ${ }^{2}$, Inne H M Borel Rinkes ${ }^{1}$ and Gerlof D Valk ${ }^{3}$ \\ Departments of ${ }^{1}$ Surgical Oncology and Endocrine Surgery, ${ }^{2}$ Anesthesiology and ${ }^{3}$ Endocrinology, University Medical Center Utrecht, Hp.nr. G04.228, \\ Heidelberglaan 100, 3584CX Utrecht, The Netherlands
}

(Correspondence should be addressed to A Scholten at Department of Surgery, University Medical Center Utrecht; Email: a.scholten-5@umcutrecht.nl)

\begin{abstract}
Objective: Hemodynamic (HD) instability still underlies difficulties during pheochromocytoma resection. Little is known about HD instability in patients with multiple endocrine neoplasia (MEN) type 2-related pheochromocytoma. Our aim was to assess differences in HD during pheochromocytoma resection between MEN2 and non-MEN patients. In addition, we sought to identify risk factors for intraoperative HD instability.

Design: Retrospective cohort study.

Methods: A total of 22 MEN2 and 34 non-MEN patients underwent 61 pheochromocytoma resections at the University Medical Center Utrecht between 2000 and 2010. All MEN2-related pheochromocytomas were diagnosed by annual screening. HD instability was assessed by measuring the frequency of hypotensive (mean arterial blood pressure (MABP) $<60 \mathrm{mmHg}$ ) and/or hypertensive (systolic arterial blood pressure (SABP) $>200 \mathrm{mmHg}$ ) episodes.

Results: Compared with non-MEN patients, MEN2 patients were younger at diagnosis, had less symptoms, lower hormone levels, and smaller tumors. Intraoperatively, MEN2 patients had a similar frequency of hypertensive episodes ( 1.3 vs $1.9, P=0.162,95 \%$ confidence interval (CI): -6.7 to 35.4 ) and a similar maximum SABP (200 vs $220 \mathrm{mmHg}, P=0.180,95 \% \mathrm{CI}:-9.7$ to 50.5$)$. However, MEN2 patients experienced less frequent (1.04 vs 2.6, $P=0.003,95 \%$ CI: 0.57 to 2.6 ) and less severe hypotensive episodes after tumor resection (lowest MABP: 52.5 vs $45.6 \mathrm{mmHg}, P=0.015,95 \%$ CI: -12.6 to 1.16). Tumor size was an independent risk factor for HD instability for the total group after multivariate analysis.

Conclusion: MEN2 patients with pheochromocytoma, despite their smaller tumors, do not distinguish themselves from non-MEN patients in terms of hypertensive episodes during pheochromocytoma resection. Therefore, pretreatment with $\alpha$ - and $\beta$-blockade remains the standard of care in MEN2related as well as in non-MEN-related pheochromocytomas.
\end{abstract}

European Journal of Endocrinology 165 91-96

\section{Introduction}

Pheochromocytomas are rare neuroendocrine catecholamine-secreting tumors, occurring mainly in the adrenal medulla $(1,2)$. Pheochromocytomas can occur as part of a familial syndrome and are, in that case, often diagnosed by periodic screening of known mutation carriers. Pheochromocytomas can present with symptoms or as an incidental mass on radiological imaging studies. Symptoms include episodic hypertension, headaches, and palpitations. Hypertensive crisis may develop in some patients that can result in myocardial infarction, cerebral vascular accident, renal failure, acute respiratory distress syndrome, and/or mortality (3).

Surgical resection is the treatment of choice for pheochromocytoma, although the surgical procedure itself can be life threatening due to hypertensive crises and multiorgan failure or profound hypotension after tumor resection $(4,5)$. During induction of anesthesia or surgical manipulation of their tumors, patients with pheochromocytoma may have wide swings in blood pressure (BP) and heart rate (HR) (6). Administration of $\alpha-, \beta-$, and/or calcium channel blockers is assumed to lower the risk of intraoperative hemodynamic (HD) instability (including preventing a hypertensive crisis), although randomized, controlled trials are lacking $(2,7,8)$.

Preoperative medication and improvements in surgical and anesthetic techniques have nearly diminished the risk of perioperative mortality associated with pheochromocytoma resection $(3,6)$. However, intraoperative HD fluctuations can lead to serious morbidity (9). Recently, high plasma norepinephrine concentration, tumor size larger than $4 \mathrm{~cm}$, a high mean arterial $\mathrm{BP}(\mathrm{MABP})$ at presentation and after $\alpha$-blockade, and more profound 
postural BP reduced after $\alpha$-blockade were identified as risk factors for HD instability during pheochromocytoma resection (10).

Literature on perioperative care of patients undergoing adrenalectomy for pheochromocytoma is often outdated and historical without considering the improvements in perianesthetic care mentioned earlier (11). Moreover, the perioperative course concerning HD data of multiple endocrine neoplasia type 2 (MEN2) patients with pheochromocytomas has typically been reported only in conjunction with sporadic cases and patients with other familial syndromes or has included only small numbers of patients $(7,12-14)$.

Because of their early identification using the annual screening of mutation carriers, we questioned whether MEN2-related pheochromocytomas are associated with less HD instability during pheochromocytoma resection. Therefore, we assessed differences in intraoperative HD between MEN2 and non-MEN patients in a large cohort. In addition, we sought to identify risk factors for intraoperative HD instability.

\section{Subjects and methods}

We took the opportunity of a large database of patients for pheochromocytoma resection, at the University Medical Center Utrecht (UMCU) in The Netherlands from January 2000 to August 2010 following a homogeneous anesthetic and surgical care, to investigate whether patients with MEN have different intraoperative HD compared with non-MEN patients. A total of 56 patients were considered for this investigation after selection from the pathology database in which the pathology results of all operatively removed tissues are included.

In our institution, the diagnosis of pheochromocytoma is based on the urinary laboratory results of catecholamines and metanephrines and the presence of an (extra-)adrenal tumor on imaging. MEN2 is defined as the presence of an MEN2 mutation in the RET gene. All pheochromocytomas in MEN2 patients were diagnosed using (annual, biochemical) screening.

$\alpha$-Blockers (doxazosin) are administered to all patients at least 2 weeks before operation. $\beta$-Blockers (metoprolol) are administered to a subgroup of patients with a HR above 80 beats/min after adequate $\alpha$-blockade. Patients are encouraged to hydrate themselves well and a salty diet is advised. Patients are admitted 1 week before surgery to maximize the dose of doxazosin and to start saline infusion 2 days before surgery. Criteria for efficacy include a systolic arterial BP (SABP) below 140-160 mmHg and a HR below 80 beats/min. Anesthetic care includes propofol, rocuronium, sufentanil and/or isoflurane, or enflurane. Intraoperatively, BP and HR values are automatically, continuously, and digitally recorded by invasive measurement through an arterial line in the radial artery. Central venous pressure is measured in all patients. Undesirable elevations in $\mathrm{BP}$ or $\mathrm{HR}$ during surgery are treated with i.v. doses of nitrates, phentolamine, and/or esmolol. Crystalloids or colloids are infused and (nor)epinephrine, phenylephrine, and/or ephedrine are administered in case of hypotension after tumor removal.

Variables investigated included patient demographics, urinary hormone levels, tumor size on preoperative imaging (computed tomography (CT) or magnetic resonance imaging (MRI)), and preoperative blockade regimes. The outcomes of hormone level were adjusted by generating a ratio of the highest level for that hormone divided by the corresponding upper limit of normal. In addition, maximum and minimum SABP, diastolic arterial BP (DABP) and MABP, and HR throughout surgery were studied. The MABP was calculated by dividing the sum of the SABP and two times the DABP by three. To measure HD fluctuations, the number of episodes that the SABP was above $200 \mathrm{mmHg}$, chosen as cutoff value for intraoperative hypertension, was scored. In addition, hypotensive complications were measured according to the number of episodes that the MABP was below $60 \mathrm{mmHg}$. Intraoperative tachycardia and bradycardia were defined as an HR above 100 and below 45 beats/min respectively.

\section{Statistical analysis}

MEN2 patients were compared with the non-MEN patients regarding patients' demographics, diseaseand treatment-related features, and regarding differences in outcome of HD instability. In addition, patients' demographics, urinary catecholamine levels, and tumor size, among other variables, were correlated with intraoperative BP fluctuations.

All data were analyzed with SPSS version 16.0 (SPSS, Inc., Chicago, IL, USA). Independent samples $t$-test was used for comparisons between groups. Pearson's correlation coefficient was used to correlate variables ( $r$ value). Multivariate linear regression analysis was used to adjust for confounding factors. Statistical significance was shown at $P<0.05$.

\section{Results}

Between January 2000 and August 2010, total of 56 patients underwent 61 resections for pheochromocytoma. Almost all patients $(n=52)$ were operated by the same surgeon (I B R). Among those, five patients underwent bilateral adrenalectomy because of bilateral pheochromocytoma. Our study population consisted of $39 \%$ MEN2 patients.

\section{MEN versus non-MEN}

Patient and tumor/diagnostic characteristics for the MEN2 group versus the non-MEN group are shown in 
Table 1 Characteristics of MEN vs non-MEN patients

\begin{tabular}{|c|c|c|c|}
\hline & MEN & Non-MEN & $\boldsymbol{P}$ value $(95 \% \mathrm{Cl})$ \\
\hline \multicolumn{4}{|l|}{ Patient characteristics } \\
\hline Patients, $n$ (\% of total population) & $22(39)$ & $34(61)$ & \\
\hline Classification, $n$ & 21 MEN2A, 1 MEN2B & $\begin{array}{l}26 \text { Sporadic, } 3 \text { VHL, } \\
3 \text { SDHD, } 2 \text { Neuro- } \\
\text { fibromatosis }\end{array}$ & \\
\hline Age at surgery (years, mean \pm S.D.) & $34 \pm 12.1$ & $46 \pm 14.3$ & 0.001 (4.74 to 19.74$)$ \\
\hline Female, $n(\%)$ & $8(36.6)$ & $17(50)$ & $0.344(-0.40$ to 0.16$)$ \\
\hline ASA physical status (mean \pm s.D.) & $2.10 \pm 0.3$ & $2.26 \pm 0.5$ & $0.128(-0.50$ to 0.39$)$ \\
\hline \multicolumn{4}{|l|}{ Co-morbidity, $n$} \\
\hline History of cardiovascular disease & 3 & 13 & $0.140(-0.25$ to 0.45$)$ \\
\hline Respiratory disease (pneumonia, COPD) & 0 & 4 & $0.444(-0.43$ to 0.56$)$ \\
\hline Endocrine disease (diabetes mellitus) & 0 & 4 & $0.444(-0.43$ to 0.56$)$ \\
\hline \multicolumn{4}{|l|}{ Tumor and diagnostic characteristics } \\
\hline Bilateral localization, $n$ & 4 & 3 & $0.32(-0.31$ to 0.10$)$ \\
\hline Hypertension at diagnosis, $n$ & 6 & 22 & $0.009(0.09$ to 0.63$)$ \\
\hline Clinical manifestations, $n$ & 9 & 23 & $<0.000(-1.23$ to -0.40$)$ \\
\hline Manifestations per patient, $n$ (mean \pm s.D.) & $2.7 \pm 2.5$ & $3.9 \pm 2.2$ & $0.065(-0.08$ to 2.54$)$ \\
\hline Cardiac manifestations, $n$ & $2--m$ & 13 & $0.010(0.07$ to 0.50$)$ \\
\hline $\begin{array}{l}\text { Highest urinary hormone ratio (multiplication } \\
\text { of reference value, mean } \pm \text { s.D.) }\end{array}$ & 4.5 & 18.1 & 0.001 (5.91 to 21.32 ) \\
\hline Metanephrine & $3.4 \pm 3.0$ & $9.4 \pm 20.1$ & $0.102(-1.25$ to 13.22$)$ \\
\hline Normetanephrine & $2.7 \pm 2.9$ & $9.7 \pm 9.9$ & $0.001(3.08 \text { to } 10.98)^{\mathrm{a}}$ \\
\hline Epinephrine & $3.9 \pm 4.2$ & $6.8 \pm 11.9$ & $0.200(-1.60$ to 7.46$)$ \\
\hline Norepinephrine & $1.2 \pm 1.6$ & $6.9 \pm 7.1$ & $<0.000(3.08 \text { to } 8.24)^{\mathrm{b}}$ \\
\hline $\begin{array}{l}\text { Largest tumor diameter on imaging }(\mathrm{cm}) \\
\text { mean } \pm \text { s.D. (range) }\end{array}$ & $2.6 \pm 1.7(1.0$ to 7.7$)$ & $5.2 \pm 2.6(1.3$ to 12.0$)$ & $<0.000$ (13.51 to 36.62$)$ \\
\hline Time symptoms to diagnosis (days, mean \pm s.D.) & $56 \pm 154$ & $559 \pm 952$ & 0.007 (140.8 to 831.91$)$ \\
\hline Time symptoms to surgery (days, mean \pm s.D.) & $226 \pm 162$ & $651 \pm 939$ & 0.021 (65.5 to 749.51$)$ \\
\hline Time diagnosis to surgery (days, mean \pm s.D.) & $175 \pm 117$ & $92 \pm 79$ & $0.003(-138.7$ to -30.94$)$ \\
\hline
\end{tabular}

MEN, multiple endocrine neoplasia; VHL, Von Hippel-Lindau syndrome; SDHD, mutation in succinate dehydrogenase B, C, and D; COPD, chronic obstructive pulmonary disease. Independent sample $t$-test was used for comparisons between groups.

Table 1. Significant differences between both groups included a younger mean age at diagnosis, less (cardiac) symptoms, lower preoperative urinary hormone levels, and a smaller tumor size on preoperative imaging for MEN2 patients.

Preoperative differences between MEN2 and non-MEN patients in terms of BP and HR (Table 2) were diminished after preoperative medication (Table 3). Anesthesia was comparable between both groups (data not shown). Intraoperatively, MEN2 patients were similar to nonMEN patients in terms of hypertension, i.e. the number of hypertensive episodes, the maximum SABP, and the number of interventions needed to treat undesirable elevations in SABP. In contrast, MEN2 patients experienced less frequent and less severe hypotensive episodes after tumor resection (Table 2). The differences between MEN2 and non-MEN patients did not change after exclusion of patients with Von Hippel-Lindau syndrome (VHL), mutation in succinate dehydrogenase B, C, and D (SDHD), or neurofibromatosis (data not shown).

There were no significant differences between the two groups regarding postoperative course (Table 4).

\section{Correlations with tumor size}

The average tumor size based on imaging studies for the total group was $4.2 \mathrm{~cm}$ (range $1-12 \mathrm{~cm}$ ). We found a correlation between tumor size and preoperative urinary hormone levels $(r=0.64, P<0.000)$. There were weak correlations between tumor size and the number of (cardiac) symptoms and the presence of hypertension at diagnosis $(r=0.29, P=0.025 ; r=0.26$, $P=0.050$ respectively).

Correlations of tumor size and systolic BP were significant regarding highest SABP at tumor manipulation $(r=0.39, P=0.002)$ and lowest SABP at tumor resection $(r=0.497, P<0.000)$ and hypertensive $(r=$ $0.50, P<0.000)$ and hypotensive episodes $(r=0.60$, $P<0.000)$. Tumor size also correlated with the number of interventions of the anesthesiologist, i.e. the frequency of using antihypertensive medications during tumor manipulation $(r=0.40, P=0.002)$ and need for vasopressors after tumor resection $(r=0.66$, $P<0.000)$. These correlations were also significant in multivariate analysis after adjustment for familial syndrome status, preoperative BP and HR, preoperative urinary hormone levels, pre-treatment regimes, and laparoscopic or open surgery.

The correlation with variables associated with arterial BP was strongest for tumors larger than $3 \mathrm{~cm}$. A tumor diameter larger than $3 \mathrm{~cm}$ was accompanied by significantly more hypertensive and hypotensive episodes compared with a tumor diameter of $3 \mathrm{~cm}$ or less $(2.02$ vs $0.47, P<0.000 ; 2.51$ vs $0.83, P<0.000$ 
Table 2 MEN vs non-MEN patients: hemodynamic data.

\begin{tabular}{|c|c|c|c|}
\hline & MEN & Non-MEN & $\boldsymbol{P}$ value $(95 \% \mathrm{Cl})$ \\
\hline \multicolumn{4}{|l|}{$\mathrm{SABP}^{\mathrm{a}}(\mathrm{mmHg}$, mean \pm S.D. $)$} \\
\hline At diagnosis & $137 \pm 22$ & $157 \pm 34$ & 0.014 (4.1 to 35.2$)$ \\
\hline After preoperative medication & $125 \pm 16$ & $126 \pm 18$ & $0.782(-7.6$ to 10.0$)$ \\
\hline Pre-start ${ }^{\mathrm{t}}$ & $139 \pm 20$ & $146 \pm 17$ & $0.101(-1.5$ to 16.8$)$ \\
\hline After induction & $93 \pm 19$ & $87 \pm 20$ & $0.287(-15.5$ to 4.7$)$ \\
\hline During tumor manipulation & $191 \pm 48$ & $204 \pm 62$ & $0.382(-17.7$ to 42.8$)$ \\
\hline After tumor resection & $94 \pm 23$ & $75 \pm 23$ & $0.001(-31.5$ to -8.1$)$ \\
\hline \multicolumn{4}{|l|}{ Intraoperative hypertension } \\
\hline Hypertensive episodes (mean \pm s.D.), $n$ & $1.3 \pm 1.4$ & $1.9 \pm 2.0$ & $0.162(-6.7$ to 35.4$)$ \\
\hline Maximum SABP (mmHg, mean \pm s.D.) & $200 \pm 57$ & $220 \pm 55$ & $0.180(-9.7$ to 50.5$)$ \\
\hline $\begin{array}{l}\text { Interventions needed to treat undesirable } \\
\text { elevations in SABP (mean } \pm \text { s.D.), } n\end{array}$ & $11.4 \pm 9.1$ & $14.8 \pm 18.8$ & $0.439(-5.31$ to 12.1$)$ \\
\hline \multicolumn{4}{|l|}{ Intraoperative hypotension } \\
\hline Hypotensive episodes (mean \pm s.D.), $n$ & $1.04 \pm 1.2$ & $2.6 \pm 2.6$ & $0.003(0.57$ to 2.6$)$ \\
\hline Minimum MABP (mmHg, mean \pm s.D.) & $52.5 \pm 10$ & $45.6 \pm 12$ & $0.015(-12.6$ to -1.16$)$ \\
\hline $\begin{array}{l}\text { Interventions needed to treat undesirable } \\
\text { decreases in MABP (mean } \pm \text { s.D.), } n\end{array}$ & $1.9 \pm 3.8$ & $5.1 \pm 5.0$ & 0.007 (0.9 to 5.6$)$ \\
\hline
\end{tabular}

respectively). A larger tumor led to more hypotensive episodes after tumor resection. Although the correlation between hypertensive episodes and tumors larger than $3 \mathrm{~cm}$ is high, small tumors can also lead to HD instability and especially to hypertension during surgery.

\section{Discussion}

Due to considerable improvements in preoperative medical preparation and perioperative anesthetic control, mortality after pheochromocytoma resection is rare. However, (morbidity from) intraoperative HD instability remains a problem. The perioperative HD course of MEN2 patients with a pheochromocytoma has typically been reported only in case reports $(6,10,12$, 14). We report results of a large cohort study comparing patients with MEN2-related pheochromocytoma with non-MEN patients with pheochromocytoma. We mainly demonstrated that MEN2 patients with pheochromocytoma do not distinguish themselves from sporadic cases of pheochromocytoma in terms of intraoperative hypertensive episodes. In addition, we also report results on hypotension during pheochromocytoma resection, where others have only focused on rises in BP and hypertensive crisis. Importantly, reduced BP associated with reduced plasma catecholamine release after tumor resection is the major cause of death. Furthermore, we demonstrated that after multivariate analysis, tumor size is an independent risk factor for HD instability.

Clinically, pheochromocytoma in MEN2 patients differs from sporadic pheochromocytoma because they are often identified at an earlier stage because of annual screening of known mutation carriers. In most cases, earlier diagnosis leads to the identification of smaller tumors, often associated with fewer symptoms and less often and less severe hypertension. In our study, indeed $70 \%$ of the MEN2 patients were normotensive and only $43 \%$ had symptoms. Cardiovascular symptoms associated with pheochromocytoma occurred in two of our MEN2 patients. These results are in agreement with previous studies $(12,15)$.

However, despite preoperative differences between MEN2 and non-MEN patients, BP after preoperative medication and intraoperative HD instability in terms

Table 3 MEN vs non-MEN patients: (pre)operative treatment regimes.

\begin{tabular}{llll}
\hline & MEN & Non-MEN & $P$ value (95\% Cl) \\
\hline Duration of $\alpha$-blockade (days), mean \pm s.D. $(n)$ & $74 \pm 50(22)$ & $48 \pm 42(33)$ & $0.047(-51.91$ to -0.37$)$ \\
Daily dosage doxazosin (mg), mean \pm s.D. $(n)$ & $17 \pm 12(22)$ & $24 \pm 20(31)$ & $0.048(0.11$ to 20.43$)$ \\
Duration of $\beta$-blockade (days), mean \pm s.D. $(n)$ & $115 \pm 150(10)$ & $56 \pm 97(16)$ & $0.269(-164.8$ to 48.12$)$ \\
Additional antihypertensive, $n$ & 1 & 12 & $0.005(0.09$ to 0.47$)$ \\
Preoperative saline infusion, $n$ & 11 & 20 & $0.647(-0.22$ to 0.35$)$ \\
Preoperative admission days (mean \pm s.D.) & $3.5 \pm 3.3$ & $3.7 \pm 3.4$ & $0.833(-1.68$ to 2.10$)$ \\
Laparoscopic surgery, $n$ & 20 & 17 & $0.004(-0.59$ to -0.12$)$
\end{tabular}

Independent sample $t$-test was used for comparisons between groups. 
Table 4 MEN vs non-MEN patients: postoperative data.

\begin{tabular}{|c|c|c|c|}
\hline & MEN, $n=22$ & Non-MEN, $n=34$ & $\boldsymbol{P}$ value $(95 \% \mathrm{Cl})$ \\
\hline Postoperative admission days (mean \pm s.D.) & $4.8 \pm 2.2$ & $6.6 \pm 4.7$ & $0.136(-0.46$ to 3.30$)$ \\
\hline Total admission days (mean \pm s.D.) & $8.3 \pm 3.8$ & $10.3 \pm 6.2$ & $0.243(-1.14$ to 4.40$)$ \\
\hline IC admission days, mean \pm s.D. $(n)$ & $1.4 \pm 1.4(7)$ & $1.5 \pm 1.7(8)$ & $0.437(-1.52$ to 1.63$)$ \\
\hline \multicolumn{4}{|l|}{ Postoperative complications, $n$} \\
\hline Tension, heart rate or fluid related ${ }^{a}$ & 3 & 6 & $0.649(-0.18$ to 0.24$)$ \\
\hline Cerebrovascular events & 0 & 0 & - \\
\hline Mortality & 0 & 0 & _- \\
\hline Otherb & 1 & 4 & $0.389(-0.09$ to 0.23$)$ \\
\hline Malignant pheochromocytoma, $n$ & 0 & 1 & $0.437(-0.05$ to 0.11$)$ \\
\hline
\end{tabular}

Independent sample $t$-test was used for comparisons between groups.

ancluding hypertension during the first postoperative days requiring additional antihypertensive, direct postoperative hypotension requiring vasopressors (mainly norepinephrine) and intravascular fluid therapy, atrial fibrillation, pulmonary edema, and cardial stunning (caused by relative cardiomyopathy secondary to the pheochromocytoma in combination with intraoperative fluid therapy).

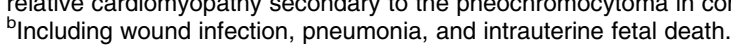

of rises in BP were similar in both groups. This might mean that the relatively small MEN2-related pheochromocytomas are easily provoked to secrete catecholamines during resection. In contrast with rises in BP, MEN2 patients experienced less frequent and less severe hypotensive episodes. These differences between groups were independent of doxazosin doses on the day of surgery.

Preoperative treatment in our study involved doxazosin in combination with metoprolol if indicated. In all of our patients, a mean SABP of $125 \mathrm{mmHg}$ was obtained after blockade. Multivariate analysis in our study demonstrated that the titrated doses of doxazosin administered preoperatively was not related to SABP after tumor resection $(P=0.927)$, indicating that in our patients higher $\alpha$-blocker doses did not lead to a more profound decrease in BP after tumor removal. Bruynzeel et al. (10) and Prys-Roberts \& Farndon (16) found similar results for doxazosin and phenoxybenzamine in terms of controlling arterial pressure and HR before and during surgery, but doxazosin caused fewer undesirable side effects both before and after surgery (16). Theoretically, patients with pheochromocytoma have a reduced intravascular volume owing to catecholamine-mediated vasoconstriction $(17,18)$. Patients in our study, therefore, received i.v. saline infusion therapy 2 days before surgery and a salty diet if they had tachycardia.

A recent report demonstrated a correlation between tumor size and HD instability (SABP above $160 \mathrm{mmHg}$ ) during pheochromocytoma resection (10), although others failed to demonstrate tumor size to be a risk factor $(19,20)$. The results of our study confirm that tumor size may be correlated with HD instability. However, we found a cutoff size of $3 \mathrm{~cm}$ instead of $4 \mathrm{~cm}$ (11) for significant association (SABP > $200 \mathrm{mmHg}$ ), independent of preoperative hormone levels, preoperative medication, surgical approach, and the presence of a familial syndrome. This correlation, as stated, is independent of surgical approach. However, surgical skill in handling the tumor can be a risk factor in intraoperative HD instability (19). In our study, almost all patients were operated by the same surgeon, which makes surgical skill less likely to play a significant role in differences in intraoperative HD between patient groups.

Previous studies have demonstrated a direct relationship between tumor size and hormone levels in plasma and urine (21-23). Preoperative urinary hormone levels in our study also correlated with tumor size and to an extent intraoperative hypertension, as an independent risk factor for HD instability. The latter is also shown in the literature $(12,24)$. Our MEN2 patients had the highest urinary hormone ratio for epinephrine compared with (nor)metanephrine and norepinephrine. They also had significantly less norepinephrine secretion compared with our non-MEN patients. This is in agreement with previous studies $(25,26)$.

A limitation of our study is its retrospective design. Therefore, patients could not be randomized for different pretreatment regimes. However, our preoperative treatment protocol makes no distinction between familial cases of pheochromocytoma and sporadic cases. This is confirmed by the fact that dosage of $\alpha$-blockade is not a confounder in identifying risk factors for HD instability. Because all HD data and use of medication during pheochromocytoma resection were recorded automatically, continuously, and digitally, only few data were missing. We used the hormone ratio to account for the differences in type of urinary hormone excreted in highest amount by the patient. Despite this, accurate correlation between tumor size and hormone level may still be affected. CT and MRI were used for preoperative imaging, which can result in size measurement variations. In addition, multiple radiologists interpreted the preoperative imaging scans, producing operator variation in final size determination.

\section{Conclusion}

Despite earlier diagnosis and significantly smaller tumors, MEN2 patients with pheochromocytoma do not distinguish themselves from sporadic cases of pheochromocytoma in terms of intraoperative hypertensive episodes. Therefore, pretreatment with $\alpha$-blockade 
started at least 2 weeks before surgery in combination with $\beta$-blockade, if tachycardia is present, remains important; MEN2 patients or patients with small tumors are not excluded.

\section{Declaration of interest}

The authors declare that there is no conflict of interest that could be perceived as prejudicing the impartiality of the research reported.

\section{Funding}

This research did not receive any specific grant from any funding agency in the public, commercial or not-for-profit sector.

\section{References}

1 Desmonts JM \& Marty J. Anaesthetic management of patients with phaeochromocytoma. British Journal of Anaesthesia $1984 \mathbf{5 6}$ 781-789. (doi:10.1093/bja/56.7.781)

2 Bravo EL \& Gifford RW. Current concepts. Pheochromocytoma: diagnosis, localization and management. New England Journal of Medicine 1984311 1298-1303. (doi:10.1056/NEJM1984111 53112007)

3 Lenders JW, Eisenhofer G, Mannelli M \& Pacak K. Phaeochromocytoma. Lancet 2005366 665-675. (doi:10.1016/S0140-6736 (05)67139-5)

4 Lo CY, Lam KY, Wat MS \& Lam KS. Adrenal pheochromocytoma remains a frequently overlooked diagnosis. American Journal of Surgery $2000 \mathbf{1 7 9}$ 212-215. (doi:10.1016/S0002-9610(00) 00296-8)

5 Apgar V \& Papper EM. Pheochromocytoma: anesthetic management during surgical treatment. Archives of Surgery $195162634-648$.

6 Feldman JM, Blalock JA, Fagraeus L, Miller JN, Farrell RE \& Wells SA Jr. Alterations in plasma norepinephrine concentration during surgical resection of pheochromocytoma. Annals of Surgery 1978188 758-768. (doi:10.1097/00000658-197812000-00 008)

7 Kinney MA, Narr BJ \& Warner MA. Perioperative management of pheochromocytoma. Journal of Cardiothoracic and Vascular Anesthesia 200216 359-369. (doi:10.1053/jcan.2002.124150)

8 Miura Y \& Yoshinaga K. Doxazosin: a newly developed, selective alpha 1-inhibitor in the management of patients with pheochromocytoma. American Heart Journal $1988 \mathbf{1 1 6} 1785-1789$. (doi:10.1016/0002-8703(88)90230-X)

9 Shupak RC. Difficult anesthetic management during pheochromocytoma surgery. Journal of Clinical Anesthesia 199911 247-250. (doi:10.1016/S0952-8180(99)00014-8)

10 Bruynzeel H, Feelders RA, Groenland TH, van den Meiracker AH, van Eijck CH, Lange JF, de Herder WW \& Kazemier G. Risk factors for hemodynamic instability during surgery for pheochromocytoma. Journal of Clinical Endocrinology and Metabolism 201095 678-685. (doi:10.1210/jc.2009-1051)

11 Goldstein RE, O'Neill JA Jr, Holcomb GW III, Morgan WM III, Neblett WW III, Oates JA, Brown N, Nadeau J, Smith B, Page DL, Abumrad NN \& Scott HW Jr. Clinical experience over 48 years with pheochromocytoma. Annals of Surgery $1999 \quad \mathbf{2 2 9} \quad 755-764$ (discussion 764-6). (doi:10.1097/00000658-199906000-00001)

12 Luo A, Guo X, Yi J, Ren H, Huang Y \& Ye T. Clinical features of pheochromocytoma and perioperative anesthetic management. Chinese Medical Journal 2003116 1527-1531.

13 Van Heerden JA, Sheps SG, Hamberger B, Sheedy PF, Poston JG \& ReMine WH. Pheochromocytoma: current status and changing trends. Surgery 198291 367-373.
14 Hamilton BP, Landsberg B \& Levine RJ. Measurement of urinary epinephrine in screening for pheochromocytoma in multiple endocrine neoplasia type II. American Journal of Medicine 197842 2928-2934.

15 Atallah F, Bastide-Heulin T, Soulié M, Crouzil F, Galiana A, Samii K \& Virenque C. Haemodynamic changes during retroperitoneoscopic adrenalectomy for phaeochromocytoma. British Journal of Anaesthesia 200186 731-733. (doi:10.1093/bja/86.5.731)

16 Prys-Roberts C \& Farndon JR. Efficacy and safety of doxazosin for perioperative management of patients with pheochromocytoma. World Journal of Surgery 200226 1037-1042. (doi:10.1007/ s00268-002-6667-z)

17 Iijima T, Takagi T \& Iwao Y. An increased circulating blood volume does not prevent hypotension after phaeochromocytoma resection. Canadian Journal of Anaesthesia 200451 212-215. (doi:10. 1007/BF03019097)

18 Stenstrom G \& Kutti J. The blood volume in phaeochromocytoma patients before and during treatment with phenoxybenzamine. Acta Medica Scandinavica 1985218 381-387. (doi:10.1111/ j.0954-6820.1985.tb08862.x)

19 Weismann D, Fassnacht M, Weinberger F, Hamelmann W, Diehl S, Lorenz K, Baerlehner E, Reincke M, Beuschlein F, Knoefel W, Nies C, Hahner S \& Allolio B. Intraoperative haemodynamic stability in patients with phaeochromocytoma - minimally invasive vs conventional open surgery. Clinical Endocrinology 200665 352-358. (doi:10.1111/j.1365-2265.2006.02603.x)

20 Plouin PF, Duclos JM, Soppelsa F, Boublil G \& Chatellier G. Factors associated with perioperative morbidity and mortality in patients with pheochromocytoma: analysis of 165 operations at a single center. Journal of Clinical Endocrinology and Metabolism 200186 1480-1486. (doi:10.1210/jc.86.4.1480)

21 Guerrero MA, Schreinemakers JM, Vriens MR, Suh I, Hwang J, Shen WT, Gosnell J, Clark OH \& Duh QY. Clinical spectrum of pheochromocytoma. Journal of the American College of Surgeons 2009209 727-732. (doi:10.1016/j.jamcollsurg.2009.09.022)

22 Huynh TT, Pacak K, Brouwers FM, Abu-Asab MS, Worrell RA, Walther MM, Elkahloun AG, Goldstein DS, Cleary S \& Eisenhofer G. Different expression of catecholamine transporters in pheochromocytomas from patients with von Hippel-Lindau syndrome and multiple endocrine neoplasia type 2. European Journal of Endocrinology 2005153 551-563. (doi:10.1530/eje.1. 01987)

23 Eisenhofer G, Walther MM, Huynh TT, Li ST, Bornstein SR, Vortmeyer A, Manelli M, Goldstein DS, Linehan WM, Lenders JW \& Pacak K. Pheochromocytomas in von Hippel-Lindau syndrome and multiple endocrine neoplasia type 2 display distinct biochemical and clinical phenotypes. Journal of Clinical Endocrinology and Metabolism 2001 86 1999-2008. (doi:10.1210/jc.86.5.1999)

24 Kinney MA, Warner ME, van Heerden JA, Horlocker TT, Young WF Jr, Schroeder DR, Maxson PM \& Warner MA. Perianesthetic risks and outcomes of pheochromocytoma and paraganglioma resection. Anesthesia and Analgesia $2000911118-1123$.

25 Eisenhofer G, Huynh T, Elkahloun A, Morris JC, Bratslavsky G, Linehan WM, Zhuang Z, Balgley BM, Lee CS, Mannelli M, Lenders JW, Bornstein SR \& Pacak K. Differential expression of the regulated catecholamine secretory pathway in different hereditary forms of pheochromocytoma. American Journal of Physiology. Endocrinology and Metabolism 2008295 E1223-E1233. (doi:10.1152/ajpendo.90591.2008)

26 Eisenhofer G, Pacak K, Huynh TT, Qin N, Bratslavsky G, Linehan WM, Mannelli M, Friberg P, Grebe SK, Timmers HJ, Bornstein SR \& Lenders JW. Catecholamine metabolomic and secretory phenotypes in phaeochromocytoma. Endocrine-Related Cancer 201018 97-111. (doi:10.1677/ERC-10-0211)

Received 2 April 2011

Accepted 15 April 2011 